\title{
Metabolic Syndrome and the Risk of Preclinical Heart Failure: Insights after 17 Years of Follow-Up from the STANISLAS Cohort
}

\author{
Abhinav Sharma ${ }^{a, b}$ Amir Razaghizad ${ }^{b}$ João Pedro Ferreira ${ }^{a, c}$ \\ Jean-Loup Machu ${ }^{\mathrm{a}} \quad$ Erwan Bozec $^{\mathrm{a}} \quad$ Nicolas Girerd $^{\mathrm{a}} \quad$ Patrick Rossignol $^{\mathrm{a}}$ \\ Faiez Zannad $^{a}$ \\ aINSERM CIC-P 1433, CHRU de Nancy, INSERM U1116, FCRIN INI-CRCT (Cardiovascular and Renal Clinical Trialists), \\ Université de Lorraine, Nancy, France; ${ }^{\mathrm{b}}$ Division of Cardiology, McGill University Health Centre, Montreal, QC, \\ Canada; 'Department of Physiology, University of Porto, Porto, Portugal
}

\section{Keywords}

Metabolic syndrome · Heart failure Preclinical heart failure

\begin{abstract}
Background: We used data from people initially free of clinical cardiovascular disease to evaluate the association between metabolic syndrome (MS) and incident preclinical heart failure (pHF). Methods and Results: STANISLAS was a familial, single-center, longitudinal prospective cohort study composed of 1,006 families from Nancy, France (median follow-up, 17 years [1993-2016]). Age- and sex-adjusted logistic regression and inverse probability weighting models were used to evaluate the association between MS and pHF, which was defined by diastolic dysfunction, atrial enlargement, ventricular hypertrophy, or elevated natriuretic peptides. Among 944 people who were adults at the first and final visit, those with baseline MS were more likely to be older (63 vs. 61 vs. 59 years of age) and male (73\% vs. 55\% vs. $45 \%)$ compared to people who developed incident MS and people who had no baseline MS, respectively. Furthermore, compared to people without baseline MS, the risk of pHF was numerically larger among people with baseline MS (adjusted odds ratio [aOR] 2.27, 95\% Cl: 1.07-4.81) and people who developed incident MS (aOR 1.56, 95\% Cl: 1.00-2.43). Con-
\end{abstract}

cerning the metabolic determinants of MS, the risk of pHF was most elevated in people with baseline hypertension (aOR 3.19, 95\% Cl: 1.80-5.63) and elevated waist circumference (aOR 2.59, 95\% Cl: 1.47-4.57). Conclusion: Overall, HF is an important public health concern given the high risk of mortality when patients with MS or elevated fasting glucose become established with the disease. Early aggressive lifestyle modification and medical intervention among patients free of cardiovascular disease with an obese-hypertensive phenotype may be warranted to prevent HF development.

(c) 2022 The Author(s).

Published by S. Karger AG, Basel

\section{Introduction}

There is a high prevalence of metabolic syndrome (MS) among patients with preclinical heart failure (HF) [1]. Although these diseases are often coprevalent, the association between MS and the risk of incident preclinical HF is unclear. Our group previously described an association between elevated waist circumference and an increased risk of diastolic dysfunction development [2]. The findings suggested that targeting MS or its metabolic determinants through lifestyle or medical intervention could be an effective strategy to decrease
Karger@karger.com www.karger.com/crd

Karger $\stackrel{\text { '⿳亠丷厂 }}{ }$

BOPEN ACCESS
(C) 2022 The Author(s)

Published by S. Karger AG, Basel

This article is licensed under the Creative Commons Attribution 4.0 International License (CC BY) (http://www.karger.com/Services/ OpenAccessLicense). Usage, derivative works and distribution are permitted provided that proper credit is given to the author and the original publisher.
Correspondence to:

Faiez Zannad, f.zannad@chu-nancy.fr 
Fig. 1. Consort diagram for the STANIS-

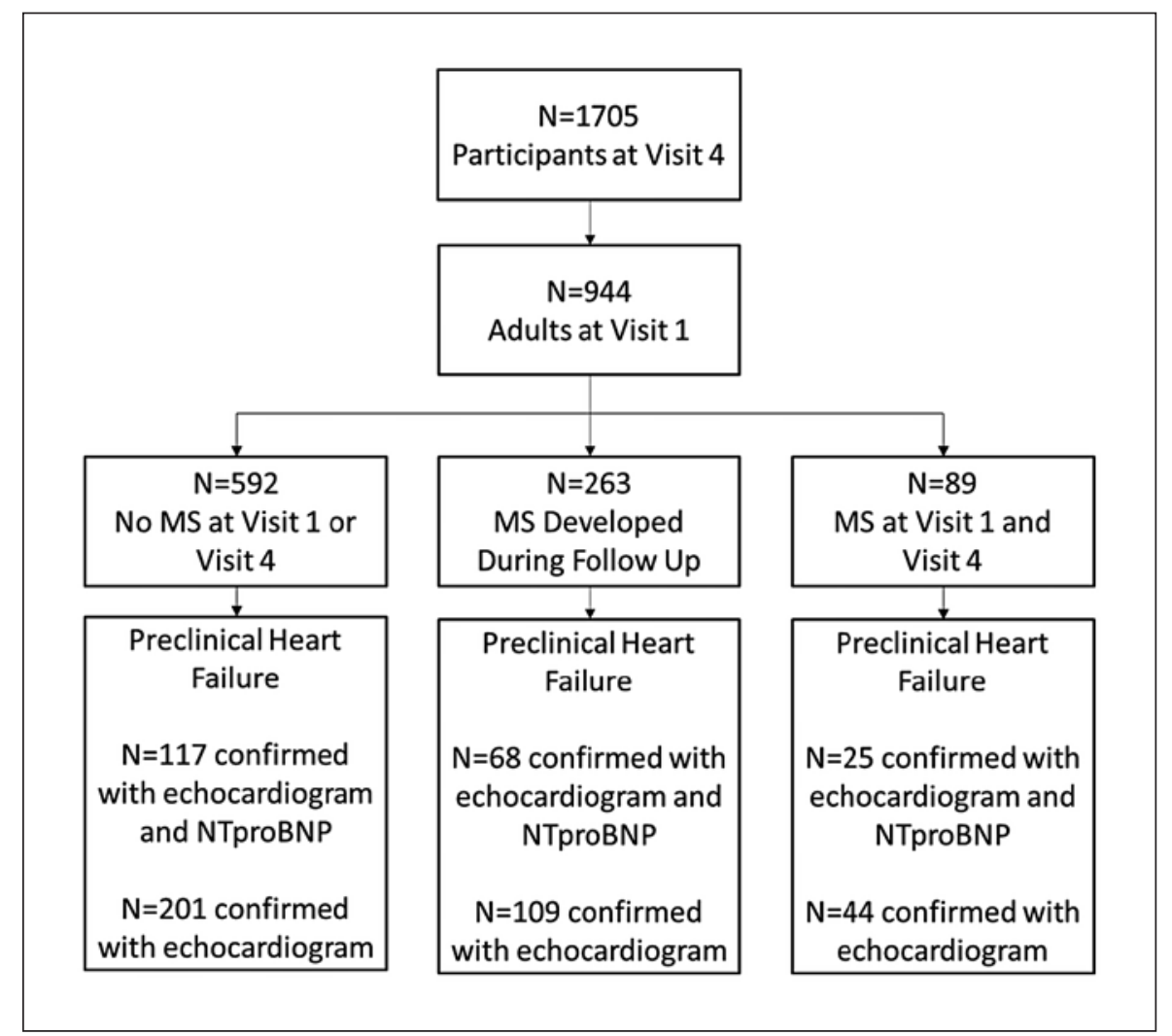
LAS cohort.

the incidence of preclinical HF. To address this knowledge gap, we used data from participants initially free of cardiovascular disease in the STANISLAS cohort (Suivi Temporaire Annuel Non-Invasif de la Santé des Lorrains Assurés Sociaux: NCT01391442) to evaluate the epidemiological association between baseline or incident MS [3], and its metabolic determinants, on preclinical HF as defined by the American Heart Association.

\section{Methods}

STANISLAS was designed as a familial, single-center, prospective longitudinal cohort study composed of 1,006 families from Nancy, France, who were recruited between 1993 and 1995. The families were healthy and free of any acute and/or chronic illnesses at recruitment. From 2011 to 2016, STANISLAS participants were revisited for a fourth examination (V4) at our department as previously described [4]. Echocardiographic assessments were conducted at the baseline (V1) and V4 examination, and biological biomarkers were routinely assessed.

Preclinical HF was defined at the V4 examination by the presence of diastolic dysfunction [5,6], left atrial enlargement, left ventricular hypertrophy, or elevated N-terminal pro-brain natriuretic peptides (NTproBNP) $>125 \mathrm{pg} / \mathrm{mL}$. This definition of preclinical HF captures echocardiographic structural features and biomarkers (i.e., NTproBNP) that have been demonstrated to increase the risk of incident $\mathrm{HF}[7,8]$.

We defined MS at the V1 and V4 examination based on the International Diabetes Federation's definition. The International Diabetes Federation defines people with MS as individuals with an elevated waist circumference ( $\geq 94 \mathrm{~cm}$ for men; $\geq 80 \mathrm{~cm}$ for women) and two of the following: raised triglycerides (>150 mg/dL [1.7 $\mathrm{mmol} / \mathrm{L}])$, reduced HDL-C $(<40 \mathrm{mg} / \mathrm{dL}[1.0 \mathrm{mmol} / \mathrm{L}]$ in $\mathrm{men} ;<50$ $\mathrm{mg} / \mathrm{dL}[1.3 \mathrm{mmol} / \mathrm{L}]$ in females; or on therapy for reduced HDLC), hypertension (systolic $\geq 130 \mathrm{~mm} \mathrm{Hg}$; diastolic $\geq 80 \mathrm{~mm} \mathrm{Hg}$; or on antihypertensive treatment), and elevated fasting glucose $(\geq 100$ $\mathrm{mg} / \mathrm{dL}$ [5.6 mmol/L]; or on treatment for elevated glucose) [3].

For the primary analysis, we evaluated the association between MS and preclinical HF among individuals who were adults at the V1 examination. We utilized age- and sex-adjusted logistic regression and inverse probability weighting models. That is, among patients at V4, the inverse probability of treatment (i.e., exposure) was computed with a propensity score that was based on a logistic model adjusted for age and sex. The inverse probability of treatment was then balanced across the three groups (i.e., baseline MS, developed MS, and absent MS) to minimize between-group clinical differences and ensure exchangeability. The association between the three groups and preclinical HF was then evaluated utilizing a generalized logistic regression model. Sensitivity analyses removing the NTproBNP criterion from the preclinical HF definition were also conducted. A global $p$ value comparing the three groups' associations with preclinical HF was computed in a multinomial weighted regression model. All analyses were conducted with SAS (v9.0). 
Table 1. Characteristics of the STANISLAS population based on metabolic syndrome status at the final visit

\begin{tabular}{|c|c|c|c|c|}
\hline Characteristics & $\begin{array}{l}\text { No metabolic syndrome } \\
\text { at baseline or follow-up } \\
(n=592)\end{array}$ & $\begin{array}{l}\text { Incident metabolic syndrome } \\
\text { developed during follow-up } \\
(n=263)\end{array}$ & $\begin{array}{l}\text { Metabolic syndrome at } \\
\text { baseline and follow-up } \\
(n=89)\end{array}$ & $p$ value \\
\hline \multicolumn{5}{|l|}{ Clinical characteristics } \\
\hline Age, years & $59(56-62)$ & $61(58-64)$ & $63(59-65)$ & $<0.0001$ \\
\hline Women, $n(\%)$ & $328(55.4)$ & $118(44.9)$ & $24(27.0)$ & $<0.0001$ \\
\hline Height, m & $1.67 \pm 0.09$ & $1.67 \pm 0.09$ & $1.70 \pm 0.09$ & 0.020 \\
\hline Weight, kg & $68(60-78)$ & $81(72-89)$ & $93(80-104)$ & $<0.0001$ \\
\hline $\mathrm{BMI}, \mathrm{kg} / \mathrm{m}^{2}$ & $24.4(22.4-26.8)$ & $28.4(26.3-31.1)$ & $31.9(28.8-34.9)$ & $<0.0001$ \\
\hline Waist circumference, $\mathrm{cm}$ & $87 \pm 11$ & $99 \pm 10$ & $110 \pm 12$ & $<0.0001$ \\
\hline Current smoker, $n(\%)$ & $73(12.3)$ & $30(11.4)$ & $10(11.2)$ & 0.93 \\
\hline $\mathrm{eGFR}, \mathrm{mL} / \mathrm{min} / 1.73 \mathrm{~m}^{2}$ & $92(82-98)$ & 90 (79-97) & $89(77-98)$ & 0.18 \\
\hline eGFR $<60 \mathrm{~mL} / \mathrm{min} / 1.73 \mathrm{~m}^{2}, n(\%)$ & $9(1.5)$ & $6(2.3)$ & $3(3.4)$ & 0.42 \\
\hline Total cholesterol, g/L & $2.27 \pm 0.35$ & $2.17 \pm 0.40$ & $1.96 \pm 0.45$ & $<0.0001$ \\
\hline $\mathrm{HDL}, \mathrm{g} / \mathrm{L}$ & $0.63 \pm 0.14$ & $0.53 \pm 0.12$ & $0.47 \pm 0.10$ & $<0.0001$ \\
\hline $\mathrm{LDL}, \mathrm{g} / \mathrm{L}$ & $1.45 \pm 0.31$ & $1.35 \pm 0.34$ & $1.19 \pm 0.37$ & $<0.0001$ \\
\hline Hypercholesterolemia treatment, $n$ (\%) & $58(9.8)$ & $125(47.5)$ & $51(57.3)$ & $<0.0001$ \\
\hline Fasting glycemia, $\mathrm{g} / \mathrm{L}$ & $0.88(0.83-0.94)$ & $0.98(0.89-1.05)$ & $1.04(0.95-1.20)$ & $<0.0001$ \\
\hline Diabetes, $n(\%)$ & $8(1.4)$ & $34(12.9)$ & $25(28.4)$ & $<0.0001$ \\
\hline Diabetes treatment, $n(\%)$ & $5(0.8)$ & $24(9.1)$ & $20(22.5)$ & $<0.0001$ \\
\hline Antihypertensive treatment, $n(\%)$ & $93(15.7)$ & $139(52.9)$ & $70(78.7)$ & $<0.0001$ \\
\hline Office SBP, $\mathrm{mm} \mathrm{Hg}$ & $125 \pm 14$ & $135 \pm 16$ & $138 \pm 17$ & $<0.0001$ \\
\hline Office DBP, mm Hg & $73 \pm 8$ & $77 \pm 9$ & $77 \pm 9$ & $<0.0001$ \\
\hline Nocturnal SBP, mm Hg & $110 \pm 10$ & $115 \pm 11$ & $118 \pm 12$ & $<0.0001$ \\
\hline Diurnal SBP, $\mathrm{mm} \mathrm{Hg}$ & $124 \pm 10$ & $128 \pm 11$ & $132 \pm 12$ & $<0.0001$ \\
\hline 24-h SBP, mm Hg & $119 \pm 10$ & $123 \pm 10$ & $127 \pm 12$ & $<0.0001$ \\
\hline NTproBNP, pg/mL* & $54(34-83)$ & $51(30-120)$ & $38(27-121)$ & 0.59 \\
\hline \multicolumn{5}{|l|}{ Echocardiographic parameters } \\
\hline LVEF, \% & $65 \pm 6$ & $66 \pm 6$ & $65 \pm 8$ & 0.23 \\
\hline LV mass/BSA, $\mathrm{g} / \mathrm{m}^{2}$ & $74(64-88)$ & $82(69-93)$ & $88(73-100)$ & $<0.0001$ \\
\hline $\mathrm{LAV} / \mathrm{BSA}, \mathrm{mL} / \mathrm{m}^{2}$ & $22(17-27)$ & $23(18-28)$ & $23(18-28)$ & 0.053 \\
\hline E wave, $\mathrm{cm} / \mathrm{s}$ & $65 \pm 14$ & $66 \pm 16$ & $67 \pm 14$ & 0.41 \\
\hline A wave, $\mathrm{cm} / \mathrm{s}$ & $64 \pm 15$ & $72 \pm 17$ & $76 \pm 15$ & $<0.0001$ \\
\hline Septal Ea velocity, cm/s & $9.0 \pm 2.3$ & $8.1 \pm 2.3$ & $7.6 \pm 1.9$ & $<0.0001$ \\
\hline Lateral Ea velocity, $\mathrm{cm} / \mathrm{s}$ & $11.4 \pm 3.2$ & $10.5 \pm 2.7$ & $9.6 \pm 2.7$ & $<0.0001$ \\
\hline Deceleration time, ms & $218 \pm 52$ & $217 \pm 60$ & $230 \pm 58$ & 0.12 \\
\hline A wave duration, ms & $137 \pm 27$ & $143 \pm 30$ & $144 \pm 23$ & 0.004 \\
\hline Pulmonary reversal A wave duration, ms & $109 \pm 20$ & $111 \pm 19$ & $114 \pm 17$ & 0.072 \\
\hline
\end{tabular}

Continuous variables are described as mean \pm SD or as median (IQR) according to their normality, and categorical variables are described as frequencies (percentage). Preclinical HF (1) was defined only on patients with an NTproBNP measurement. Subjects had a preclinical HF if they had DD grade II or III and/or LVH and/or LAE and/or high NTproBNP. Preclinical HF (2) was defined in all the population and excluding the NTproBNP criterion. Subjects had preclinical HF if they had DD grade II or III and/or LVH and/or LAE. BMI, body mass index; eGFR, estimated glomerular filtration rate; HDL, high-density lipoprotein; LDL, low-density lipoprotein; SBP, systolic blood pressure; DBP, diastolic blood pressure; LVEF, left ventricular ejection fraction; LV, left ventricular; LAV, left atrial volume; LVH, left ventricular hypertrophy; LAE, left atrial enlargement. $* n=447$ with NTproBNP measurements.

Biological samples were obtained by venipuncture at the Nancy Hospital Center's Centre d'Investigation Clinique. Using sample-handling protocols, serum and plasma samples were obtained. All samples were then preserved at temperatures ranging from $-80^{\circ} \mathrm{C}$ to $-196^{\circ} \mathrm{C}$ in a central biobank. NTproBNP was then quantified utilizing an electrochemiluminescence immunoassay (Elecsys System, Roche Diagnostics, Basel, Switzerland).

Metabolic Syndrome and Preclinical Heart Failure

\section{Results}

The median follow-up time was 17 years (1993-2016). Among the 1,705 participants with V4 examinations between 2011 and 2016, only 944 individuals were adults at the V1 examination (Fig. 1). Among these 944 individu- 


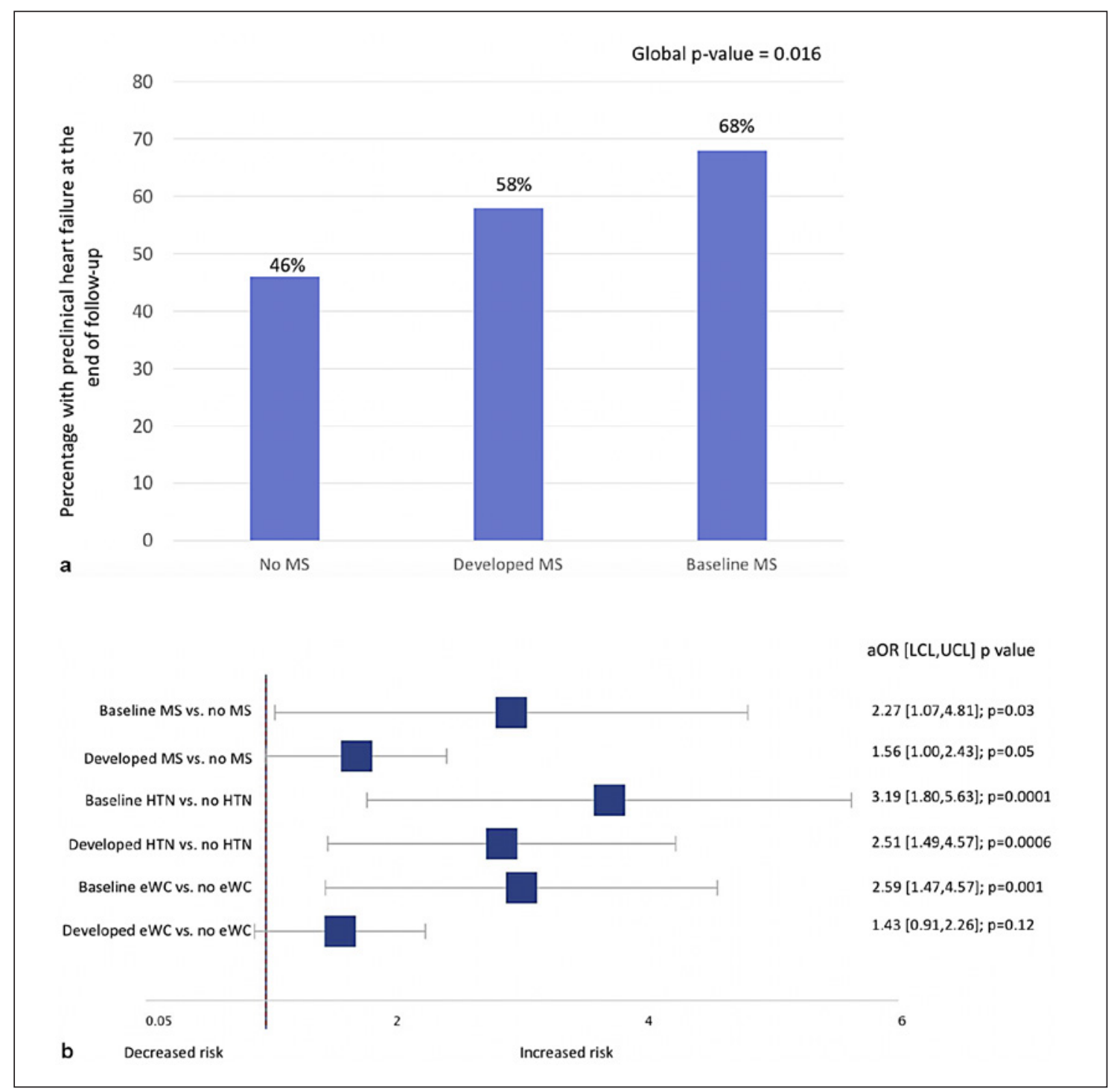

Fig. 2. a Presence of preclinical HF at the end of follow-up. b aOR for the risk of preclinical HF at the end of follow-up. MS, metabolic syndrome; HTN, hypertension; eWC, elevated waist circumference; aOR, adjusted odds ratio; LCL, lower confidence limit; UCL, upper confidence limit.

als, 872 (or $92 \%$ of the adult population) had echocardiograms conducted at V4, and of this subpopulation, 408 people had available data for NTproBNP at the same visit.

Overall, 89 (9\%) patients had baseline MS and 263 (28\%) patients developed MS during follow-up. At the V1 examination, $5 \%$ of study participants had a BMI $>30 \mathrm{~kg} /$ $\mathrm{m}^{2}$. At the V4 examination, study participants with baseline MS were more likely to be older (63 vs. 61 vs. 59 years of age), male ( $73 \%$ vs. $55 \%$ vs. $45 \%)$, have elevated waist circumference (110 vs. 99 vs. $87 \mathrm{~cm})$, hypertension $(79 \%$ vs. $53 \%$ vs. $16 \%)$, and have elevated fasting glucose $(28 \%$ vs. $13 \%$ vs. $1.4 \%$; Table 1 ) compared to participants who developed MS and participants who had no MS at baseline, respectively.

In total, 210 of the 408 (51.4\%) patients with complete echocardiographic and biomarker data developed preclinical HF (Fig 1). Compared to patients without baseline MS, the risk of preclinical HF was numerically increased both among patients who developed incident MS (adjusted odds ratio [aOR] 1.56, 95\% CI: 1.00-2.43, $p=$ 0.05 ) and patients who had MS at baseline (aOR 2.27, 95\% 
CI: $1.07-4.81, p=0.03$; Fig. 2). Regarding the metabolic determinants of MS, the risk of preclinical HF was elevated in individuals with hypertension (aOR 3.19, 95\% CI: $1.80-5.63$ ) and elevated waist circumference (aOR 2.59, 95\% CI: 1.47-4.57; Fig. 2). However, on evaluation, the other metabolic determinants of MS including elevated triglycerides, high-density lipoprotein, or fasting glucose were not associated with preclinical HF.

In the sensitivity analysis, 354 of the 872 (40.6\%) patients developed preclinical HF with respect to the definition that omitted NTproBNP. Consistent results for the risk of preclinical HF were seen in this sensitivity analysis. Compared to patients without baseline MS, the risk of preclinical HF was numerically increased both among patients who developed incident MS (aOR 1.29, 95\% CI: $0.94-1.78, p=0.11)$ and among patients who had MS at baseline (aOR 2.27, 95\% CI: 1.07-2.81, $p=0.04$ ). Adjusting for BMI in addition to age and sex attenuated the significant association of baseline MS and preclinical HF (aOR 1.15; 95\% CI: 0.49-2.71; $p=0.75$ ), but a numerically increased risk was still observed.

\section{Discussion}

After a median of 17 years of follow-up in our cohort of patients initially free of cardiovascular disease, baseline MS and incident MS were shown to be associated with an increased risk of preclinical HF. In addition, chronic exposure to an elevated waist circumference and hypertension (reflecting an overweight-hypertensive phenotype) appeared to be the metabolic determinants most associated with preclinical HF development.

Our analysis extends on prior work from cohorts with older patients with greater degrees of cardiovascular comorbidities. A prior analysis of 51,451 patients in the Women's Health Initiative, Multi-Ethnic Study of Atherosclerosis (MESA), and CHS suggested a dose-response relationship between BMI and the risk of HF with preserved ejection fraction ( $p$ trend HR <0.0001) [9]. In the MESA study, the risk of future cardiovascular disease (including clinically confirmed HF) was increased in patients with baseline obesity who developed MS compared to individuals without elevated BMI (aOR 1.60; 95\% CI: 1.14-2.25) [10]. The combination of core laboratory-reported echocardiograms within a homogeneous patient cohort and long length of follow-up supports a robust assessment of the role of MS in the genesis of preclinical HF.

Our study had some limitations. First, our analysis did not assess the impact of diet and physical activity on mod-

Metabolic Syndrome and Preclinical Heart Failure ifying the relationship between MS and preclinical HF. As a result, the impact of diet and physical activity on HF development warrants further evaluation. Second, prior analyses have identified a poor association between biomarkers (e.g., NTproBNP) and structural heart disease; as a result, the use of natriuretic peptides in our definition of preclinical HF may have limited the analysis. Nevertheless, when we removed NTproBNP from the definition, the association between baseline MS and preclinical HF was still observed [11]. Third, the use of indexed left atrial volume or left ventricular mass, especially among patients with obesity, may have underestimated the presence of preclinical HF. Likewise, among patients who have atrial fibrillation or who are athletes, the prevalence of preclinical HF could have been overestimated. Fourth, the number of participants who had available data on NTproBNP and echocardiographic data $(n=408 ; 46.8 \%)$ was smaller than the population of adult patients with echocardiographic data alone $(n=872)$. Nevertheless, the association between baseline MS and preclinical HF was consistent in sensitivity analyses where NTproBNP was removed from the preclinical HF definition. Finally, there was no further follow-up available beyond V4 to enable analyses evaluating the association between MS and clinically confirmed acute HF.

Given the high risk of mortality when patients with MS or elevated fasting glucose become established with HF [12], HF prevention remains an important public health concern. Evaluation of early aggressive lifestyle, including diet and exercise, may help prevent preclinical and eventual HF development. Dietary strategies to reduce weight, especially among individuals who are obese and hypertensive, may represent a strategy for HF prevention [13]. Furthermore, the evaluation of various medical interventions such as blood pressure control or use of sodium-glucose cotransporter-2 inhibitors [14] among patients free of cardiovascular disease with an obese-hypertensive phenotype to prevent future HF development is warranted.

\section{Conclusion}

Our study demonstrated that after 17 years of followup, patients with baseline MS, especially those with obesehypertensive phenotypes, are at increased risk of developing preclinical HF. As a result, the primary prevention of preclinical HF should be an important public health focus given the high risk of mortality when patients with metabolic aberrations are diagnosed with clinically confirmed 
HF. Evaluating the preventive efficacy of early aggressive lifestyle modification and medical intervention among patients free of cardiovascular disease with obese-hypertensive phenotypes may be warranted.

\section{Acknowledgments}

The authors would like to express their sincere gratitude to all the staff at the Clinical Investigation Center who were involved in the daily operations associated with the STANISLAS cohort.

\section{Statement of Ethics}

All STANISLAS participants consented to having their data utilized for subsequent secondary analyses. As a result, no separate ethics approval was needed for the present study. The local Ethics Committee in Nancy, France (Comité de Protection des Personnes Est III-Nancy-France) approved the initial research protocol (ClinicalTrials.gov identifier NCT01391442).

\section{Conflict of Interest Statement}

A.S. reports receiving support from the Fonds de Recherche $\mathrm{Du}$ Quebec Sante (FRQS) Junior 1 clinician scientist award, Takeda, Roche Diagnostics, BMS-Pfizer and is funded by the Alberta Innovates Health Solution Clinician Scientist Fellowship. A.R. reports receiving funding from McGill University's Graduate Excellence Award in Medicine \& Ethelwyn and Ernie Nyman Fellowship. P.R. reports receiving consulting fees from Novartis, NovoNordisk, Relypsa, AstraZeneca, Grünenthal, Idorsia, Stealth Peptides, Fresenius, and Vifor; lecture fees from Bayer and CVRx; and he is the cofounder of CardioRenal. F.Z. reports consulting fees for serving on the board of Boston Scientific; consulting fees from Novartis, Takeda, AstraZeneca, Boehringer Ingelheim, GE Healthcare, Relypsa, Servier, Boston Scientific, Bayer, Johnson \& Johnson, and Resmed; and speaking fees from Pfizer and AstraZeneca. There are no other conflicts to report.

\section{Funding Sources}

The STANISLAS study is sponsored by Nancy CHRU. This work is supported by the French Ministry of Health "Programme Hospitalier de Recherche Clinique Inter regional 2013," by the Contrat de Plan Etat-Lorraine and FEDER Lorraine, and a public grant overseen by the French National Research Agency (ANR) as part of the second "Investissements d'Avenir" program FIGHTHF (reference: ANR-15-RHU-0004), and by the French PIA project "Lorraine Université d'Excellence," reference ANR-15-IDEX04-LUE. It is also supported by the European Fibro-Targets Project (Grant agreement No. SP7\#602904), European HOMAGE project (Grant agreement No. Heart "Omics" in Ageing, 7th Framework Program Grant \# 305,507), the MEDIA project (Européen "Cooperation"-Theme "Health"/FP7-HEALTH-2010-single-stage (reference: 261409), FOCUS-MR (reference: ANR-15CE14-0032-01), ERA-CVD EXPERT (reference: ANR-16ECVD-0002-02), and the Fondation de Recherche en Hypertension Artérielle.

\section{Author Contributions}

The study was conceptualized by A.S., and F.Z. A.S. and J.P.F. contributed to the study methodology. A.S. and J.P.F. conducted the study investigation and contributed to the writing (original draft) of the manuscript. A.S., A.R., J.P.F., J.L.M., E.B., N.G., P.R., and F.Z. contributed to the writing (review and editing) of the manuscript. F.Z. supervised the study. We attest that all authors have reviewed and approved the article prior to its submission.

\section{Data Availability Statement}

The data that support the findings of this study are not publicly available because of their containing information that could compromise the privacy of research participants but are available from the corresponding author (F.Z.) upon reasonable request.

\section{References}

1 Seferović PM, Petrie MC, Filippatos GS, Anker SD, Rosano G, Bauersachs J, et al. Type 2 diabetes mellitus and heart failure: a position statement from the heart failure association of the European Society of Cardiology. Eur J Heart Fail. 2018;20(5):853-72.

2 Chau K, Girerd N, Magnusson M, Lamiral Z, Bozec E, Merckle L, et al. Obesity and metabolic features associated with long-term developing diastolic dysfunction in an initially healthy population-based cohort. Clin Res Cardiol. 2018;107(10):887-96.
3 Alberti KGMM, Eckel RH, Grundy SM, Zimmet PZ, Cleeman JI, Donato KA, et al. Harmonizing the metabolic syndrome: a joint interim statement of the international diabetes federation task force on epidemiology and prevention; National heart, lung, and blood institute; American heart association; World heart federation; International Atherosclerosis Society; and International Association for the Study of Obesity. Circulation. 2009; 120(16):1640-5.
4 Ferreira J, Girerd N, Bozec E, Merckle L, Pizard A, Bouali S, et al. Cohort profile: rationale and design of the fourth visit of the STANISLAS cohort: a familial longitudinal population-based cohort from the Nancy region of France. Int J Epidemiol. 2018;47(2): 395-j.

5 Nagueh SF, Smiseth OA, Appleton CP, Byrd BF, Dokainish H, Edvardsen T, et al. Recommendations for the evaluation of left ventricular diastolic function by echocardiography: an update from the American Society of Echocardiography and the European Association of Cardiovascular Imaging. J Am Soc Echocardiogr. 2016 Apr;29(4):277-314. 
6 Huttin O, Fraser AG, Coiro S, Bozec E, Selton-Suty C, Lamiral Z, et al. Impact of changes in consensus diagnostic recommendations on the echocardiographic prevalence of diastolic dysfunction. J Am Coll Cardiol. 2017; 69(25):3119-21.

7 Ammar KA, Jacobsen SJ, Mahoney DW, Kors JA, Redfield MM, Burnett JC, et al. Prevalence and prognostic significance of heart failure stages. Circulation. 2007 Mar;115(12):156370.

8 Shah AM, Claggett B, Loehr LR, Chang PP, Matsushita K, Kitzman D, et al. Heart failure stages among older adults in the community: the atherosclerosis risk in communities study. Circulation. 2017;135(3):224-40.
9 Pandey A, LaMonte M, Klein L, Ayers C, Psaty BM, Eaton CB, et al. Relationship between physical activity, body mass index, and risk of heart failure. J Am Coll Cardiol. 2017;69(9): 1129-42.

10 Mongraw-Chaffin M, Foster MC, Anderson CAM, Burke GL, Haq N, Kalyani RR, et al. Metabolically healthy obesity, transition to metabolic syndrome, and cardiovascular risk. J Am Coll Cardiol. 2018;71(17):1857-65.

11 Nah EH, Kim SY, Cho S, Kim S, Cho HI. Plasma NT-proBNP levels associated with cardiac structural abnormalities in asymptomatic health examinees with preserved ejection fraction: a retrospective cross-sectional study. BMJ Open. 2019;9(4):e026030.
12 Sharma A, Demissei BG, Tromp J, Hillege HL, Cleland JG, O'Connor CM, et al. A network analysis to compare biomarker profiles in patients with and without diabetes mellitus in acute heart failure. Eur J Heart Fail. 2017; 19(10):1310-20.

13 Ebbeling CB, Feldman HA, Steltz SK, Quinn NL, Robinson LM, Ludwig DS. Effects of sugar-Sweetened, artificially Sweetened, and Unsweetened beverages on cardiometabolic risk factors, body composition, and sweet taste preference: a randomized controlled trial. J Am Heart Assoc. 2020 Aug 4;9(15):e015668.

14 Sharma A, Pagidipati NJ, Califf RM, McGuire DK, Green JB, Demets D, et al. Impact of regulatory guidance on evaluating cardiovascular risk of new glucose-lowering therapies to treat type 2 diabetes mellitus: lessons learned and future directions. Circulation. 2020; 141(10):843-62. 